\title{
Mise en scène du moi érotique, variations autobiographiques et jeux littéraires dans Journal intime de Lydia Vázquez
}

\author{
Staging of the erotic self, autobiographical variations \\ and literary games in Lydia Vázquez's Journal intime
}

\author{
Mathilde Tremblais \\ Doctora por la Universidad del País Vasco \\ mathilde.tremblais@hotmail.com
}

\begin{abstract}
This article proposes an interest in the work of Lydia Vázquez, Journal intime (2019), a text of an autobiographical nature that is fully within the scope of erotic literature. The rhetoric typical of the writings of the I that presents the story will be studied and both the narrative structure and the formal and discursive modalities will give rise to reflection. A preferential space will be dedicated to the analysis of the autobiographical pact that the author establishes with her reader. The specificity of female eroticism will be dealt with through the vision that the author offers of an eroticism that tends towards the artistic expression of post-pornography. The aim of this article is to show the singularity that distinguishes Lydia Vázquez's work in the landscape of contemporary French-language erotic literature.
\end{abstract}

\section{Keywords}

story analysis, ego writings, autobiographical pact, literary theory, eroticism

\begin{abstract}
Resumen
Este artículo propone interesarse por la obra de Lydia Vázquez, Journal intime (2019), un texto de carácter autobiográfico que se inscribe plenamente en el ámbito de la literatura erótica. La retórica propia de las escrituras del yo que presenta el relato será estudiada y tanto la estructura narrativa como las modalidades formales y discursivas darán lugar a una reflexión. Se dedicará un espacio preferente al análisis del pacto autobiográfico que la autora establece con su lector. La especificidad del erotismo femenino será tratada a través de la visión que la autora ofrece de un erotismo que tiende hacia la expresión artística de la post-pornografía. El objetivo de este artículo es mostrar la singularidad que distingue la obra de Lydia Vázquez en el paisaje de la literatura erótica contemporánea de lengua francesa.
\end{abstract}

\section{Palabras clave}

análisis del relato, escrituras del yo, pacto autobiográfico, teoría literaria, erotismo 


\section{Introduction}

Lydia Vázquez est une auteure d'origine espagnole, essayiste et critique littéraire. Ses travaux portent principalement sur la littérature libertine dont elle est spécialiste. Elle est aussi la traductrice en espagnol de Crébillon fils, Sade, Pierre Louÿs ou Apollinaire, entre autres écrivains. Dans le domaine de la littérature contemporaine, elle a signé, pour l'éditeur Cabaret Voltaire, les traductions en espagnol de certaines œuvres d'Abdellah Taïa, d'Annie Ernaux ou d'Annie Le Brun. Journal intime est la dernière œuvre littéraire de Lydia Vázquez, publiée aux Éditions Du Murmure en juillet 2019, elle est illustrée par Miguel Ángel Martín, un écrivain et dessinateur de renom.

Journal intime est un texte à caractère autobiographique qui s'inscrit pleinement dans le registre de la littérature érotique. Plusieurs perspectives pouvaient être adoptées pour analyser le journal de Lydia Vázquez, nous avons ici choisi de privilégier l'étude de l'écriture du moi érotique, d'en dégager les spécificités à travers plusieurs axes. L'attention portera d'abord sur les traits distinctifs propres à la littérature intime que présente le récit. Les modalités discursives et la structure narrative choisies par l'auteure seront abordées pour rendre compte de la particularité d'un texte qui se présente comme un journal intime. Les réflexions concernant le dispositif discursif et le caractère marginal du pacte autobiographique que Lydia Vázquez scelle avec son lecteur permettront de mieux cerner la singularité de l'écriture du moi érotique que l'auteure déploie dans son récit. Enfin, la spécificité de l'érotisme féminin que l'œuvre reflète sera observée à travers la vision que l'auteure offre d'un érotisme qui tend vers l'expression artistique de la post-pornographie. L'ensemble des analyses menées dans cet article montrera que l'écriture du moi érotique de Lydia Vázquez dans Journal intime est dotée d'une profonde originalité, contribuant à faire de ce livre intense et riche une œuvre rare dans le panorama de la littérature érotique contemporaine de langue française.

\section{Les traits distinctifs de la littérature intime}

À travers le choix du titre, Journal intime, Lydia Vázquez décide d'ancrer son texte dans le domaine très vaste que représente la littérature intime. Dès le début de son journal, elle qualifie l'exercice auquel elle va se livrer de confession: "Il faut aussi que je dise, dans ce premier jour de confession" (Vázquez, 2019: 10). À la fin de ce que l'auteure désigne ici comme son premier jour de confession, elle fait référence à “un cahier” (Vázquez, 2019: 12), l'objet matériel qui l'accompagne et sur lequel elle épanche ses émois. Dans ces lignes sur lesquelles s'achève la première partie du texte, le premier jour de confession qui sert d'introduction, l'auteure décrit son entreprise en ces termes: 
Les pages qui vont suivre donneront compte de mes ébats amoureux, de mes rêveries érotiques, de mes fantaisies sexuelles des six mois prochains. Cette réflexion liminaire ne se voulait qu'une mise au point. Le point de départ d'un cahier joyeux, jouissant, jouissif, mais aussi mélancolique, enragé, rouge et noir, mouvant. Vivant. Comme moi (Vázquez, 2019: 12).

$\mathrm{Au}$ fur et à mesure que le récit progresse, le terme "journal” tend à s'imposer dans le texte et, au milieu de l'œuvre, l'auteure précise le véritable sens de son écrit en le caractérisant de "mon journal érotique" (Vázquez, 2019: 54). La dernière partie s'achève symboliquement le $1^{\text {er }}$ mai 2015, le jour où Lydia Vázquez décide de mettre un point final à son texte. Dans ce dénouement, l'écrivaine fait de nouveau figurer les termes qui définissent son projet littéraire quand elle évoque significativement "la fin de mon journal érotique" (Vázquez, 2019: 102).

Tout le texte est traversé par des marques appartenant à la rhétorique propre aux écritures du moi. À titre d'exemple, le verbe "avouer" ou son substantif "aveu", très récurrents dans la littérature du moi, sont présents dans les pages de Journal intime: "Je prends, durant ces mois, la plume, pour avouer, car tout journal est un aveu" (Vázquez, 2019: 51) et "Ceci ne serait pas mon journal érotique si je n'y avouais pas..." (Vázquez, 2019: 54). Le souci de vérité qui anime l'acte d'écriture est palpable à travers des affirmations telles que "Mais je dois tout dire" (Vázquez, 2019: 54), ou celle où l'écrivaine exprime la volonté de transparence qui guide sa parole: "Et je dois avouer ici, espace de confession..." (Vázquez, 2019: 98). Ces citations ne vont pas sans rappeler le sens qui motive toute entreprise autobiographique, comme celle du texte fondateur que représente Les Confessions de Jean-Jacques Rousseau, un philosophe dont Lydia Vázquez est spécialiste ${ }^{1}$.

La structure narrative qui encadre le récit de Journal intime est composée de vingtneuf divisions, qui font référence aux vingt-neuf journées que l'auteure aurait décidé de raconter. Généralement, il s'agit de journées organisées autour d'un épisode représentant le plus souvent un laps de temps relativement court. Chacun des épisodes forme une unité thématique au sein du récit. Par ailleurs, il convient de souligner que le temps de l'énoncé est aisément repérable mais le temps de l'énonciation, lui, l'est nettement moins. Le lecteur ignore le plus souvent d'où l'écrivaine conduit son récit, même si parfois elle donne des pistes en évoquant des villes comme Saragosse ou Bilbao qui semblent être les lieux qui sont le décor à partir duquel s'établit le temps de l'énonciation.

Peut-être davantage que n'importe quel autre texte appartenant aux écritures du moi, le journal intime, et celui de Lydia Vázquez plus qu'un autre, suggère la problématique qu'incarne le destinataire. Même s'il s'agit d'un texte autarcique, le journal intime pose en effet la question du destinataire, aussi bien du destinataire textuel que du lecteur extratextuel. Le premier est incarné dans le journal intime de Lydia Vázquez par une figure masculine énig-

1 Lydia Vázquez est l'auteure de nombreux articles critiques portant sur l'œuvre rousseauiste et d'un essai publié chez l'éditeur Síntesis en 2005. 
matique, un homme dont le nom ne sera jamais dévoilé, pas plus que son âge, son origine ou le lieu où il réside. L'auteure postule l'existence de cet homme qui prend vie dans et par le texte de Journal intime, une œuvre qui semble, parfois, avoir été écrite pour lui. Elle fait souvent référence au personnage masculin en ayant recours à l'expression "mon homme"2. Dans la dernière partie du journal, l'homme est désigné, à deux reprises, par le pronom "lui", typographié en italique ${ }^{3}$. Peu avant la fin du texte, l'homme est de nouveau désigné par le pronom "Lui", mais qui apparaît, cette fois, anaphorique et en majuscules. Ces éléments montrent que l'homme acquiert de plus en plus d'importance au fil du récit et que sa présence, plutôt discrète au début de l'œuvre, finit par s'imposer, à tel point qu'il s'avère être celui qui a inspiré et stimulé l'acte littéraire. Et au-delà de ce destinataire textuel, Lydia Vázquez, en publiant son journal intime, un écrit qui n'est généralement pas destiné à être divulgué, s'adresse à un public potentiel, à divers lecteurs extratextuels, pour exprimer et faire connaître la singularité de son être sexuel.

Un tel projet n'est pas sans rappeler d'autres journaux intimes écrits par des auteures de littérature érotique contemporaine de langue française. Ainsi en est-il dans Stratégie d'une passion (2004), un récit de Nathalie Gassel, une écrivaine belge qui, depuis le début du XXI siècle, s'est forgé une place à part au sein du domaine très varié que forment les écritures du moi érotique. Stratégie d'une passion s'avère être un journal intime où le "je" de Nathalie Gassel se projette sur l'avant de la scène pour donner à connaître aux lecteurs la spécificité de son moi érotique. Tout comme Journal intime de Lydia Vázquez, la narratrice de Stratégie d'une passion s'adresse à un homme énigmatique qui n'est jamais nommé et qui demeure auréolé de mystère tout au long du récit. L'évocation de cette figure masculine sert à Nathalie Gassel à inventer, à travers la fiction, des variations où son moi érotique se profile, se décline et se recrée de page en page pour s'imposer finalement comme le véritable sujet d'analyse autour duquel s'articule le discours du journal intime. Dans l'incipit de Stratégie d'une passion, Nathalie Gassel prétend que l'homme auquel elle s'adresse est le moteur de l'écriture du moi érotique mais, au fur et à mesure que le récit se déroule, le personnage masculin apparaît davantage comme le prétexte que prend l'auteure pour mener une réflexion sur soi, ce qui est, par définition, le propre de tout projet qui s'inscrit dans le champ des littératures intimes. Dans Stratégie d'une passion, Nathalie Gassel se laisse ainsi aller à des divagations, souvent d'ordre philosophique, sur la nature de son moi, son désir empirique et sa soif de reconnaissance. L'écrivaine caractérise l'exercice littéraire auquel elle se livre d" "exhibitionnisme détonnant" (Gassel, 2004: 26), qui reflète "une force mégalomaniaque" (Gassel, 2019: 48) ou un "ego hypertrophié" (Gassel, 2004: 89). Dans ce sens, la présence du personnage

2 En effet, le protagoniste est désigné pour la première fois en ces termes: "Mon homme, je le perçois là, debout, de dos, devant moi, de toutes les facettes de mes yeux clos" (Vázquez, 2019: 19). Plus loin, la narratrice continue à le nommer de la même manière: "Je n'ai pas l'habitude de m'exciter avant l'arrivée de mon homme" (Vázquez, 2019: 65) et elle évoque “cet homme que j’aime, mon homme” (Vázquez, 2019: 70).

3 Nous renvoyons ici à la page 97 du récit.

4 Ce pronom, ainsi typographié, figure à la page 101 du texte. 
masculin qui semblait au départ motiver l'écriture a tendance à s'estomper, au point que le lecteur arrive à l'oublier face à l'ampleur qu'acquiert le moi de Nathalie Gassel qui se met à nu et s'expose. Dans Journal intime de Lydia Vázquez, même si l'homme traverse le récit et demeure présent jusqu'à la fin, et même s'il apparaît comme la source d'inspiration du texte, il ne s'avère pas moins être, lui aussi, le prétexte auquel la femme a recours pour s'écrire et se raconter.

Une autre caractéristique renforce l'appartenance du journal intime de Lydia Vázquez aux écritures du moi, il s'agit des dessins du personnage féminin présents dans le péritexte et à travers lesquels on peut reconnaître l'auteure. Miguel Ángel Martín attribue à la protagoniste une silhouette mince, des cheveux bruns coupés au carré et des traits fins qui rappellent ceux de Lydia Vázquez. Non seulement le péritexte souligne la ressemblance physique entre le personnage féminin et l'auteure mais le paratexte, plus précisément la couverture de l'œuvre, accentue aussi ce rapprochement. En effet, le paratexte éditorial qu'est la couverture et où figurent le titre, Journal intime, le nom de l'auteure, Lydia Vázquez, et le dessin de la protagoniste autorise à établir l'identité entre la femme telle que Miguel Ángel Martín la dessine et Lydia Vázquez elle-même, l'auteure de Journal intime.

\section{Les modalités formelles et discursives}

Comme il a été dit, Journal intime appartient au domaine des écritures du moi. Cependant, il s'agit d'un texte dont les particularités formelles et discursives méritent d'être étudiées de près. Contrairement à la plupart des journaux intimes, le texte de Lydia Vázquez ne présente pas les traits génériques du journal intime dans la mesure où il n'est pas daté ni ne reproduit les faits et gestes du quotidien. En effet, Journal intime ne possède pas les spécificités formelles propres aux journaux telles qu'elles ont été définies par les théoriciens de la littérature qui se sont intéressés à ce type textuel. Dans son essai Le journal intime, Françoise Simonet-Tenant fait remarquer la réalité protéiforme du journal et exprime la nécessité d'en offrir une définition minimale. Dans ce sens, elle observe que:

Le journal se présente sous la forme d'un énoncé fragmenté qui épouse le dispositif du calendrier et qui est constitué d'une succession d'entrées (une entrée désignant l'ensemble de lignes écrites sous une même date) (Simonet-Tenant, 2001: 11).

Et quelques lignes plus loin, dans son approche théorique sur le journal intime, Françoise Simonet-Tenant insiste sur le fait que, parmi la diversité des journaux: "Reste la présence de la date, distinction essentielle et indispensable de l'écriture journalière. Aussi les journaux sans dates de certains semblent-ils une négation même du journal" (Simonet-Tenant, 2001: 12). Les chercheurs qui se sont intéressés au journal personnel ou au journal intime ont souligné l'importance de la datation, la présence d'entrées mises en relation avec 
le calendrier (Braud, 2006: 161-162). Les auteurs de Journal intime, histoire et anthologie (2006) soutiennent que "la base du journal, c'est la date. Le premier geste du diariste est de la noter en tête de ce qu'il va écrire. [...] La datation peut être plus ou moins précise ou espacée, mais elle est capitale" (Lejeune et Bogaert, 2006: 23).

L'absence de relation datée est un argument qui permet d'affirmer que Journal intime de Lydia Vázquez n'est pas en réalité un journal. Outre le fait qu'il ne soit pas daté, force est de constater qu'il ne reproduit pas non plus les faits et gestes du quotidien. La relation journalière s'efface, laissant place à une narration qui donne l'impression que l'auteure déroule une histoire qui débuterait le 2 novembre 2014 et ne s'achèverait que le $1^{\text {er }}$ mai 2015. Lydia Vázquez retrace principalement une succession d'épisodes qui seraient compris dans ce laps de temps mais elle ne fait pas le récit fragmenté de différentes journées datées. Les conditions de l'écriture journalière ne sont pas réunies pour que la narration simultanée, bien qu'elle soit de rares fois présente ${ }^{5}$, domine l'ensemble du récit. Les divisions qui structurent le texte portent un numéro et font davantage penser, de ce fait, à des chapitres ${ }^{6}$ qu'à des journées.

De nombreux arguments incitent à penser que Journal intime relève davantage de l'autobiographie que du journal intime. L'écriture répétitive et tâtonnante qui est la caractéristique des journaux est absente du texte de Lydia Vázquez. Par ailleurs, l'œuvre comprend un récit rétrospectif porté sur un plan d'ensemble, ce qui est le propre de l'autobiographie. Dans l'incipit et les pages qui suivent, l'auteure évoque son enfance et sa famille à travers les événements marquants qu'elle a vécus pendant son jeune âge, se pliant ainsi au passage obligé propre au genre de l'autobiographie. Dans le cas de Journal intime, il faut parler d'autobiographie sexuelle car Lydia Vázquez met principalement l'accent sur l'apprentissage qu'elle fait de la sexualité. L'œuvre dégage ainsi un caractère initiatique, les premières expériences sexuelles sont narrées et représentent autant de passages obligés des autobiographies sexuelles car elles contribuent à la construction de la personnalité érotique de l'auteure.

Par ailleurs, le point de vue critique nous incite à ne pas considérer Journal intime comme une autofiction dans la mesure où l'œuvre ne comprend pas de terme générique qui indiquerait qu'il s'agit d'un roman, ni à aucun moment de son récit Lydia Vázquez n'emploie ce terme "roman" pour qualifier son projet. Vincent Colonna, spécialiste de l'autofiction et auteur de L'autofiction, essai sur la fictionnalisation de soi en littérature (1989), une thèse novatrice sur le sujet dirigée par Gérard Genette, dégage la caractérisation fonctionnelle suivante:

5 Citons l'un des rares exemples où le caractère simultané propre à l'écriture diariste est présent dans le texte: "Dans la pénombre, j'écris. Je suis chez lui. Il dort. Je me suis levée pour noter ce qui vient de se passer entre nous" (Vázquez, 2019: 46).

6 Il semble plus juste en effet de parler de chapitres et non de journées pour désigner les divisions du texte, c'est la raison pour laquelle le terme "chapitre" sera dorénavant privilégié. 
À la différence de l'autobiographie qui serait l'apanage des vies mémorables, l'autofiction serait le refuge des vies ordinaires. Elle permettrait à chacun de raconter sa vie, dès lors qu'il la dote des atouts de la fiction. [...] C'est la première raison invoquée pour justifier l'indication générique "roman" de ce texte si fortement référentiel (Colonna, 1989: 17).

Dans Journal intime, l'absence d'indication générique "roman" oblige à ne pas considérer le récit comme une autofiction, même si, curieusement, le terme "autofiction" apparaît dans l'épitexte, notamment dans le résumé qui est donné du livre et où il est défini comme une "petite autofiction érotique". Dans son texte, Lydia Vázquez n'élabore pas une fictionnalisation de son moi, aucun effet fictionnalisant ne semble être produit ou recherché par l'écrivaine. De même, aucune intention fictionnelle n'est annoncée ou suggérée qui pourrait laisser penser que l'auteure, en dépit de ce qu'elle promet, confère un statut fictif à son journal qui, contre toute attente, vise à s'éloigner du registre référentiel dans lequel il est pleinement inscrit.

Lydia Vázquez ne succombe pas à l'effet de mode qu'a suscité le type textuel qu'est l'autofiction depuis qu'il a été découvert et théorisé. Si l'on doit à Serge Doubrovsky la naissance de l'autofiction, celle-ci était pratiquée bien avant que l'écrivain ne l'expose dans Fils (1977) et il existe une autofiction avant la lettre qui n'est pas étrangère à la littérature érotique écrite par des femmes. L'originalité de Lydia Vázquez tient précisément au fait qu'elle ne cède pas à la tentation autofictionnelle à laquelle est encline la littérature contemporaine puisqu'elle s'attache au genre purement autobiographique.

Même s'il paraît plus juste de parler d'autobiographie que d'autofiction ou de journal intime pour définir l'œuvre de Lydia Vázquez, dans cet article le terme “journal” continuera d'être utilisé car c'est le terme dont se sert l'écrivaine. On peut imaginer que le titre choisi par Lydia Vázquez, Journal intime, pour caractériser une entreprise qui, d'un point de vue théorique, ne peut être désignée comme telle, est une imprécision sémantique voulue par l'auteure pour brouiller les frontières entre les genres et entre les différents modèles qui constituent la littérature intime. Après ces distinctions théoriques, il convient de se pencher sur l'une des marques d'autoréférentialité les plus significatives de tout texte à caractère autobiographique et dont Journal intime n'est pas exempt: le pacte autobiographique que noue l'écrivaine avec son lecteur.

\section{La singularité du pacte autobiographique}

Le texte de Lydia Vázquez présente un caractère autobiographique qui se renforce au fur et à mesure des pages. Comme pour la plupart des modèles d'écriture intime, le récit met en scène une voix homodiégétique et Journal intime fait ressortir la voix homodiégétique du 
personnage féminin, qui s'affirme à travers l'omniprésence du "je" de la narratrice, le "je" qui renvoie à celui de l'auteure, la femme-écrivain qui se décline au fil des pages.

Un élément essentiel au sein du texte de Lydia Vázquez permet de poser cette identité onomastique irréfutable entre l'auteure, la narratrice et la protagoniste: le pacte autobiographique. Cet élément discursif s'avère être fondamental pour identifier les écrits à caractère autobiographique. Philippe Lejeune, le principal théoricien de l'autobiographie, a mené des recherches novatrices en ce qui concerne le pacte autobiographique et les traits définitoires qu'il présente. Dans son essai Le pacte autobiographique (1975), qui demeure aujourd'hui encore la référence en la matière, Philippe Lejeune explique: “Une identité est, ou n'est pas. [...] Pour qu'il y ait autobiographie, il faut qu'il y ait identité de l'auteur, du narrateur et du personnage" (Lejeune 1975: 15). Les travaux théoriques entrepris par Philippe Lejeune constituent un champ de recherches fécond, comme en témoigne l'essai de Carole Allamand, Le "Pacte" de Philippe Lejeune ou l'autobiographie en théorie (2018).

La plupart des écrivains qui décident de se livrer à l'exercice autobiographique conçoivent un pacte autobiographique qui représente un moment fort du récit, un événement rhétorique à part entière. En général, ils créent un pacte autobiographique dès le début de la narration, de façon à renseigner sur la nature autobiographique de leur texte, à orienter l'horizon de lecture et à combler les attentes des lecteurs potentiels. Ainsi, Philippe Lejeune soutient-il que "L'autobiographie: c'est un mode de lecture autant qu'un type d'écriture" (Lejeune, 1975: 45). Le lecteur est souvent friand d'épisodes réellement vécus et racontés par un personnage public dont il deviendrait en quelque sorte le confesseur privilégié. Dans un récit qui se définit comme "une confession érotique", il est aisé d'imaginer que le lecteur, qui adopte la position de voyeur, est d'autant plus curieux de savoir si le "je" qui domine la narration correspond à celui l'auteur.

Ces considérations au sujet du pacte autobiographique et de la réception des écrits autobiographiques, font de Journal intime une œuvre autobiographique dont la spécificité mérite d'être observée. Le pacte autobiographique que Lydia Vázquez scelle avec son lecteur renferme une particularité discursive dans la mesure où l'identité onomastique entre l'auteure, la narratrice et la protagoniste ne s'établit que tard dans le récit, plus précisément dans le dernier tiers du livre, à l'inverse de la plupart des textes autobiographiques et, bien plus, des récits contemporains qui se définissent comme des confessions érotiques.

En effet, les écrivaines classées comme auteures érotiques qui se sont livrées à l'exercice autobiographique établissent un pacte autobiographique avec le lecteur dès l'incipit de leur texte ou parfois même avant, dans une introduction ou un avertissement au lecteur qui permet de renseigner sur le registre autobiographique qui détermine le texte et oriente l'horizon de lecture. Dans ce sens, Françoise Rey, auteure d'une œuvre foisonnante et considérée dans la littérature française contemporaine comme la grande dame de l'érotisme, choisit souvent de sceller un pacte autobiographique avec son lecteur dès la préface, où elle déclare, de 
manière solennelle, la dimension autobiographique dont est imprégné son récit. Tel est le cas de Souvenirs lamentables, dont l'histoire est précédée d'un avertissement dans lequel l'écrivaine s'interroge sur le sens de son titre, sur les enjeux de l'écriture du moi qu'elle pratique et tisse avec son lecteur un pacte autobiographique.

Dans les œuvres érotiques de Françoise Rey, le pacte autobiographique est souvent renforcé par l'inscription des textes dans un espace autobiographique plus vaste où les récits se correspondent et se complètent. Ainsi, certains des textes érotiques de Françoise Rey configurent un riche espace autobiographique, c'est le cas des quatre récits suivants: La Femme de papier (1989), La Gourgandine (2002), Souvenirs lamentables (2002) et Vers les hommes (2004). Ces narrations sont parsemées de clins d'œil autobiographiques qui renvoient les unes aux autres, comme les allusions à "la Femme de papier" qui désignent Françoise Rey elle-même, l'auteure de ce texte sulfureux, La Femme de papier (1989), sa première œuvre érotique, celle qui l'a fait connaître et qui, avec Le Boucher (1988) d'Alina Reyes, a marqué un tournant dans la littérature érotique contemporaine écrite par des femmes.

Comme le fait Françoise Rey, Catherine Millet offre dans son récit La Vie sexuelle de Catherine M. (2001) un autre exemple de pacte autobiographique explicite, et d'autant plus explicite que le titre même éclaire la nature du texte. En effet, le prénom et l'initiale du nom de l'auteure compris dans le titre ne laissent pas planer le doute dans l'esprit du lecteur qui sait, avant même de commencer la lecture, que Catherine Millet décide dans son livre de raconter sa vie sexuelle. Le prologue qui précède le récit et dans lequel l'auteure entreprend sa justification renforce le pacte autobiographique que présente le titre, un pacte d'autant plus frappant que l'auteure décide de l'établir dès la couverture même de son livre en faisant apparaître une photo d'elle nue. Certains des pactes que Nathalie Gassel élabore sont aussi directs que celui de Catherine Millet et s'établissent de manière aussi frontale. L'écrivaine belge utilise tout autant le paratexte que le péritexte pour illustrer ses récits érotiques par des autoportraits où elle pose en exhibant son corps hors norme?

Ces comparaisons servent à dégager la spécificité de Journal intime, qui, lui, présente un pacte autobiographique beaucoup plus discret que ceux qui sont contenus dans les œuvres de Françoise Rey, Catherine Millet ou Nathalie Gassel. De plus, dans le texte de Lydia Vázquez, ce n'est pas la narratrice elle-même qui décline son identité mais un autre personnage qui la révèle. Ainsi, dans l'un des épisodes centraux de l'œuvre, la coiffeuse dévoile au lecteur que la protagoniste porte le nom de Lydia ${ }^{8}$. Il s'agit du seul instant du récit où le nom apparaît, le nom de famille, lui, n'étant jamais mentionné. Dans une narration homodiégétique qui se réclame du domaine de la littérature intime, le fait qu'une tierce personne désigne la narratrice par le prénom de l'auteure suffit pour que cet événement rhétorique qu'est le pacte autobiographique ait lieu. Au moment précis de Journal intime où le lecteur découvre que la

7 C'est le cas du récit déjà cité, Stratégie d'une passion, de Construction d'un corps pornographique (2005) et de Récit plastique (2008).

8 Ce pacte autobiographique figure à la page 86 de Journal intime. 
narratrice-protagoniste porte le prénom Lydia, comme l'auteure, se produit l'acte discursif qu'est le pacte autobiographique.

L'apparition du prénom de l'auteure à un stade aussi avancé de la narration réoriente désormais la compréhension de l'œuvre et la réinvestit d'une nouvelle signification. Le surgissement tardif d'un pacte autobiographique dans la trame du récit invite à réinterpréter l'ensemble de Journal intime, l'horizon d'attente du lecteur s'en trouvant profondément changé. À travers cet étonnant pacte autobiographique, Lydia Vázquez formule une promesse à son lecteur, elle montre sa volonté d'inscrire son propos dans une logique référentielle, dans une sorte de contrat véridique où elle s'engage à dire la réalité des expériences vécues car, comme le souligne Philippe Lejeune, "le genre autobiographique est un genre contractuel" (Lejeune, 1975: 44).

Il convient d'insister sur le fait que le moment discursif que représente le pacte autobiographique n'intervient que tard dans la narration de Journal intime. Par ce procédé, l'auteure parvient à cultiver l'ambigüité jusqu'à ce que le pacte autobiographique soit vraiment posé. Le lecteur du texte peut en effet se demander si c'est véritablement Lydia Vázquez qui se raconte dans les pages de son journal. Le titre lui-même, Journal intime, de par l'absence d'adjectif possessif, pourrait laisser entendre que l'auteure entreprend de relater les faits et gestes d'une autre personne. Les dessins du personnage féminin, sur la couverture ou dans les pages du livre, ne restent au fond que des dessins, dont la force autobiographique est moindre que s'il s'agissait de photographies.

La plupart des femmes qui ont fait le choix d'écrire leur confession sexuelle n'ont cessé de revendiquer leur nom à travers de multiples pactes autobiographiques solennels et explicites. Lydia Vázquez constitue une exception dans la mesure où elle préfère offrir un pacte autobiographique unique et singulier, qui apparaît comme une signature discrète, presque timide, qui contraste avec l'audace que manifeste son personnage féminin et le genre érotique ou pornographique dans lequel elle a choisi d'inscrire son œuvre.

\section{De l'érotisme à la post-pornographie}

Plusieurs arguments permettent d'affirmer que Journal intime appartient au genre de la littérature érotique. L'érotisme est présent dès les premiers mots du texte, où il se mêle à la mort. Cette vision de l'érotisme ne va pas sans rappeler la conception que défend Georges Bataille qui, dans son essai canonique L'Érotisme (1957), écrit que: "De l'érotisme, il est possible de dire qu'il est l'approbation de la vie jusque dans la mort" (Bataille, 2001: 17). Le philosophe de l'érotisme a illustré les thèses qu'il soutient dans ses récits où c'est la mort qui guide le comportement érotique des personnages. Différentes écrivaines se sont inspirées des textes de Georges Bataille en faisant, elles aussi, une place prépondérante à la mort dans les fictions érotiques qu'elles inventent. L'Orage (1996) de Régine Deforges est, peut-être, 
l'exemple le plus éclatant de l'influence qu'a pu jouer Bataille sur la vision de l'érotisme d'une auteure. Lydia Vázquez, dont le Journal intime n'est pas aussi bataillien que le récit de Régine Deforges, propose un érotisme qui est néanmoins profondément marqué par la mort, comme en rend compte l'incipit du récit:

Jour des morts. Éros ne saurait déambuler dans ces pages encore blanches si ce n'est de la main de Thanatos. Je suis marquée du sceau de la mort dès ma naissance. Avant moi, mon frère aîné que je n'ai jamais connu et que j'ai toujours porté en moi, Ernesto, est mort. Un tramway l'a écrasé devant chez nous. Le désespoir infini de José et d'Araceli, que je n'ai jamais appelés du nom de père et de mère, s'est transformé en rage érotique. Et de cette énergie née de la mort suis-je née moi-même. Car ce même jour j'ai été conçue (Vázquez, 2019: 9).

Ces lignes expliquent que tout au long de l'œuvre la mort est omniprésente et qu'elle donne son sens aux expériences érotiques auxquelles se livre la narratrice. Cependant, à l'inverse de Bataille pour qui l'érotisme ouvre à la mort ${ }^{9}$, Lydia Vázquez conçoit parfois l'acte érotique comme le moyen de défier la mort à laquelle elle se sait destinée. Ainsi l'auteure écrit, quand elle va à la rencontre de son homme:

Et moi, je suis toujours là. À voir passer les printemps, les uns après les autres. À mourir à petit feu. La mort s'empare de moi, m'avale à petites gorgées. S'en délecte. Jouit. Pour la narguer, je n'ai que ma jouissance. Je vole vers lui, vers ma jouissance et la sienne. Pour oublier que je meurs (Vázquez, 2019: 90).

Dans les pages de son récit, Lydia Vázquez décline des facettes de son moi érotique et toutes les explorations intimes qu'elle mène ont un sens érotique. La définition qu'elle propose d'elle-même est en ce sens particulièrement éclairante: "Je suis un être érotique" (Vázquez, 2019: 10), annonce-t-elle dès le début de son récit. Peu après, elle poursuit et dresse de sa personne le portrait d'une femme libre qui tend vers le désir: "Je me suis réclamée libre. Une femme libre et jouissive. En toute circonstance” (Vázquez, 2019: 11).

Comme tout autobiographe, et qui plus est femme et auteure de récits érotiques, Lydia Vázquez doit faire face à l'hostilité sociale que suscite un comportement sexuel comme le sien. Dans le chapitre 15, la narratrice évoque les dangers qu'encourent les femmes qui osent vivre leurs phantasmes et qui s'exposent ainsi aux réprobations les plus farouches. La métaphore filée de l'hydre que développe l'auteure dans ce chapitre, et à laquelle elle a recours pour désigner la société, sert à renforcer la menace qui pèse sur les femmes qui, comme la narratrice, se définissent comme libres et jouissives. Le texte adopte alors un caractère fantastique, palpable à travers les allusions au monstre, au fauve, à la bête ou à l'hydre qui est décrite comme "Fanatique, intolérante, superstitieuse, raciste, machiste" (Vázquez, 2019: 50-51). Cette hydre désigne le corps social qui attaque et condamne les sujets libres qui osent

9 "L'érotisme ouvre à la mort" (Bataille, 2001: 31). 
s'adonner à la sexualité en bravant les tabous et qui libèrent la parole qui s'est accoutumée à taire les choses du sexe.

Dans Journal intime, Lydia Vázquez conçoit le corps de la femme comme le lieu par excellence de l'intime. Pour elle, le corps érotique féminin qu'elle met en scène est le moteur de l'écriture et le moyen qui permet d'approcher au plus près la vérité intime de son être. Ainsi décrit-elle le sens de son geste: "Je prends, durant ces mois, la plume, pour avouer, car tout journal est un aveu, ce que j'ai de plus intime : mon corps à l'œuvre” (Vázquez, 2019: 51). S'il est vrai que les autobiographies de femmes se révèlent soucieuses de dire la spécificité du corps féminin, force est de constater que Journal intime renouvelle et subvertit les discours attendus sur le sujet.

En effet, certaines des réflexions que mène l'écrivaine peuvent, dans ce sens, surprendre. L'extase érotique est généralement considérée comme un instant éphémère, limité dans le temps. L'auteure de Journal intime semble concevoir le contraire, par exemple quand elle déclare: "Seul le moment érotique dure indéfiniment" (Vázquez, 2019: 36), ou bien quand, quelques lignes plus loin, elle compare l'orgasme à "un moment à goût d'éternité" (Vázquez, 2019: 38). Les pages du texte offrent des réflexions sur les significations de l'orgasme, comme le fragment suivant qui est dans ce sens l'un des plus évocateurs:

Cependant, pour moi l'orgasme a une charge hautement symbolique. D'affranchissement, de libération de toutes les aliénations, de toutes les chaînes, de toutes les oppressions. À l'instant même de l'orgasme, du vrai orgasme (le faux n'étant qu'au service de tout le contraire), on s'oublie comme sujet social, comme citoyen contraint à des lois, à des règles pour devenir, le temps que ça dure, un être suprême et unique, seul d'une solitude toute jubilatoire et énergique (Vázquez, 2019: 38).

La quête du plaisir érotique est un thème central de Journal intime, en effet la narratrice se décrit elle-même comme: "Un être évolué qui ne suit plus son instinct reproducteur mais qui, sciemment, cherche son plaisir, à le multiplier, à le varier, à le perpétuer" (Vázquez, 2019: 83). Par ailleurs, l'auteure n'hésite pas à offrir des réflexions sur son propre plaisir. On sait à quel point l'écriture sur soi est un défi périlleux mais cet exercice devient combien plus difficile lorsque l'auteur prétend aborder la question de son plaisir sexuel, et davantage encore si c'est une femme qui se livre à cette entreprise. Si l'évocation du plaisir sexuel revient à dévoiler l'une des facettes les plus intimes de son être, il existe un acte qui signifie encore un pas de plus dans le dévoilement de l'être intime: communiquer à partir de son imaginaire érotique. Ainsi, Lydia Vázquez glisse de l'analyse de sa conception du plaisir à l'aveu de ce qui stimule son imagination érotique, un glissement qui suggère une progression dans le processus du dévoilement de l'intime:

Mais je suis de nature tendre et je préfère des préliminaires sensuels, la gradation. Passer, comme on faisait au XVIII ${ }^{\mathrm{e}}$ siècle, par le réveil et l'excitation de tous les sens avant de jouir des plaisirs érotiques. Seulement quand la vue, l'ouïe, le goût, l'odorat, le 
toucher seront stimulés (ou "irrités" comme on disait dans ce XVIII siècle bien plus matérialiste que le nôtre), ce sixième sens qu'on appelait autrefois génésique pourra s'épanouir pleinement. Cette excitation n'étant jamais la même, c'est par cette préparation que je contribue à éveiller mon imagination, qui se charge de varier postures, attitudes, paroles et caresses... Il en va de ma santé érotique (Vázquez, 2019: 65-66).

La narratrice fait preuve d'une imagination débordante, le chapitre 24 est peut-être celui du journal qui rend le mieux compte de cette imagination sans frein qui est la sienne. Dans ce chapitre, les mains de la coiffeuse qui masse le crâne de la narratrice vont déchaîner chez cette dernière des rêveries érotiques d'une force rare. La vision d'un sexe d'homme doté de dimensions gigantesques s'impose dans l'imaginaire de la protagoniste et envahit son esprit:

De grosses veines parcourent le phallus démesurément grand, couronné par un bout tellement enflé qu'on le dirait prêt à exploser. La main de ma coiffeuse se fait plus ferme, et ses mouvements plus précis, plus réguliers, dans un va-et-vient qui scande mon corps, ravi de cahoter de plus belle grâce si je ne me retenais pas, consciente de la multitude d'yeux qui pourraient se tourner sur moi, curieux et brillants (Vázquez, 2019: 86).

Dans cette scène, comme dans de nombreux textes érotiques, un lieu public sert de décor aux phantasmes éveillés et l'érotisme s'installe dans un espace qui n'est pas destiné à accueillir des ébats réservés à la sphère intime. Quand l'Éros se glisse dans l'interdit de l'espace public, les actes décrits gagnent en intensité, comme dans cet épisode chez le coiffeur au cours duquel la narratrice laisse libre cours aux caprices de son imagination érotique. Le texte raconte ce qui ne doit pas être montré, ce qui permet d'affirmer que l'obscène n'est pas absent de la séquence narrative. Le dispositif de représentation que Lydia Vázquez met en œuvre pour narrer cet épisode érotique met le spectateur en position de voyeur et il en est de même des autres personnages qui assistent en silence à la scène. D'où l'obscénité qui caractérise cette séquence toute entière, une obscénité qui doit s'entendre telle qu'elle a été définie par Jean-Marie Goulemot:

Au-delà de la pratique à laquelle elle renvoie, l'obscénité exige des témoins, une présence extérieure exigée, une exhibition, une espèce de mise en scène destinée à rendre particulière sa perception par un regard extérieur convié certes mais demeurant étranger. Elle est à mettre en relation avec le théâtre, dont par bien des aspects elle relève (Goulemot, 1999: 5).

Il n'y a pas de grande œuvre érotique qui ne soit pas, en même temps, pornographique. Le texte de Lydia Vázquez ne cède pas à "la tentation pornographique"10 qui hante la littérature contemporaine mais appartient pleinement au genre pornographique. La dimension pornographique de Journal intime s'illustre notamment dans des descriptions très précises à

10 Nous empruntons cette expression à l'auteur de La littérature pornographique, Dominique Maingueneau (Maingueneau: 2007, 97). 
travers lesquelles l'auteure a recours à la crudité de certains mots pour désigner les choses du sexe. Lydia Vázquez manifeste dans son récit un goût très marqué pour les détails relatifs aux attributs sexuels mais pas seulement. L'œil amoureux fait ainsi l'objet d'une page, cet œil qui se transforme sous l'emprise de l'amour et se pare pour aller à la rencontre de l'autre. L'œil est un motif très exploité dans les récits érotiques, comme dans Histoire de l'œil (1928), un texte de Georges Bataille dont l'œil dessine la trame. L'auteure de Journal intime reprend ce motif mais le dote d'une nouvelle signification, en l'investissant d'un rôle amoureux.

La portée post-pornographique s'illustre à travers l'importance qui est accordée aux organes sexuels, éléments sur lesquels vient se greffer le discours érotique qu'exprime Lydia Vázquez. L'importance que les parties sexuelles revêtent est telle qu'elles apparaissent douées d'une volonté propre et qu'elles en arrivent même parfois à être les véritables protagonistes du journal, ainsi l'auteure écrit-t-elle:

Je pense à ça, à mes parties du corps qui, en effet, deviennent des êtres désirants, avec leurs envies singulières, différentes les unes des autres, parfois mêmes opposées, le tout avec ma tête à la merci de leur caprice. Mon esprit n'a qu'à se laisser entraîner par l'une ou l'autre, au risque de se faire tirailler. Mais n'est-ce pas cela que je veux, précisément? (Vázquez, 2019: 59).

Lydia Vázquez a recours à la prose poétique pour mettre en scène les attributs sexuels de la narratrice, qu'elle évoque en réinventant les formes du "je" lyrique ${ }^{11}$. Dans l'avant-dernier chapitre, elle s'identifie à chacune des parties sexuelles qu'elle examine, en commençant par "Je suis ce sexe béant", "Je suis ce clitoris titillant", "Je suis cette toison entortillée", "Je suis cet anus ombragé”, pour conclure à la même page: "Je suis tous ces plis, ces coins, ces recoins, ces chemins qui conduisent vers le néant, le gouffre, la bouche béante...” (Vázquez, 2019: 100), qui sont autant d'images de l'intimité. Cette mise en pièces du corps, décrite dans une langue érogène très subtile, est propre à l'expression artistique qui caractérise la post-pornographie. Catherine Millet se livre à cet exercice dans son récit autobiographique, elle s'adonne à une mise en pièces de son propre corps à travers des variations aux accents poétiques, comme dans ce passage:

Enfin, je me rendis à l'évidence: le clitoris n'était pas une pointe vive repérable comme un clou au mur, ou comme le clocher dans un paysage, ou comme le nez au milieu de la figure, c'était une sorte de nœud embrouillé, sans véritable forme propre, un minuscule chaos se produisant à la rencontre de deux petites langues de chair comme lorsque le ressac jette deux vagues l'une contre l'autre (Millet, 2001: 228).

Les descriptions très détaillées qu'offrent aussi bien Catherine Millet que Lydia Vázquez dans leur récit autobiographique ont pour objectif de mettre sur l'avant de la scène le

11 Notons qu'il est possible d'établir de nombreuses correspondances entre le "je" lyrique et le "je" autobiographique (Lecarme et Lecarme-Tabone, 2015: 35-40). 
corps érotique de la femme-écrivain et de faire ressortir la portée féministe qui caractérise leur discours. Ainsi, la dimension féministe qui sous-tend le geste artistique de Lydia Vázquez est palpable à travers une approche de la jouissance féminine qui se veut différente de celle qu'offre la pornographie classique et qui passe par une revendication du corps féminin. Ainsi, dans le livre de David Courbet Féminismes et pornographie (2012), la philosophe Sonia Bressler, déclare-t-elle:

Si nous devons définir une approche féministe de la pornographie : il me paraît évident que le corps est montré différemment par des femmes. Et ce quel que soit la sexualité. Les réalisatrices vont redonner à l'érotisme sa dimension mentale (la montée du désir, l'affect, l'émoi..). [...] Mais avant tout, elles vont davantage respecter la musique de la jouissance, le tempo d'une relation assumée. Elles offrent une alternance de plans entre de délicates palpitations charnelles et des instants plus crus, submersions d'émotions, enchaînements faits de déconstructions et d'ouvertures (Bressler, 2012: 228-229).

Par ailleurs, comme dans de nombreux textes qui appartiennent à la littérature érotique contemporaine, l'humour n'est pas absent du Journal intime de Lydia Vázquez. À la fin du deuxième chapitre, après avoir minutieusement raconté le plaisir sexuel qui s'empare de son être et la fait chavirer, la narratrice dévoile au lecteur que le responsable de son ravissement n'est autre qu'un escargot qui se promène sur sa fente. De même, l'humour est suggéré lorsque la narratrice relate que ses premiers attouchements sexuels sont motivés par une statuette représentant la vierge de Lourdes qui lui avait été offerte par une amie de sa mère. Cet épisode ne va pas sans rappeler une autre œuvre érotique, Entre todas las mujeres (1992), où l'auteure, Isabel Franc, met en scène une fillette qui doit elle aussi ses premières explorations sexuelles à une figurine de la Vierge de Lourdes.

Dans Journal intime, Lydia Vázquez met en place un système intertextuel particulièrement riche. Les écrivains qui ont forgé la personnalité de l'auteure traversent le récit, parfois sur le mode de l'explicite, comme Pierre Louÿs qui est cité et d'autres ne sont que suggérés, à travers de subtiles allusions à leur vie ou à leur œuvre. Ainsi, Lydia Vázquez évoque dans son texte Sade, Diderot ou Jarry, par l'explicite du citationnel ou l'implicite de l'allusif. Le dispositif intertextuel permet un jeu de miroirs, où les voix des écrivains se répondent et viennent se greffer à celle de l'auteure qui les fait émerger et les porte. L'effet créé est alors celui d'une polyphonie narrative, d'un dialogisme entre la voix homodiégétique féminine qui domine le récit de Journal intime et celle des auteurs rendus présents grâce à la stratégie intertextuelle mise en œuvre par Lydia Vázquez. Ces procédés utilisés contribuent sans aucun doute à accentuer la profonde littérarité dont est empreint Journal intime.

Le sens dans lequel nous entendons ici "littérarité" se doit d'être éclairé. Depuis 1921, année où Roman Jakobson forgea le terme de "littérarité"12 et proposa celle-ci comme objet

12 Dans une conférence prononcée en 1919 et publiée en 1921, Roman Jakobson utilise le terme literaturnost, traduit en français par "littérarité", et en offre la définition suivante: "Ainsi l'objet de la science de la littérature 
d'étude, divers chercheurs, comme Tzvetan Todorov ou Mircea Marghescou, se sont intéressés au concept de littérarité dont le statut demeure aujourd'hui encore ambigu. Dans Littérature et littérarité, un essai de mise au point (1984), Thomas Aron soutient que "la littérarité est une notion introuvable" (Aron, 1984: 20) et souligne les discussions qu'elle peut parfois générer ${ }^{13}$. Pour tenter d'aborder cette notion problématique, Thomas Aron suggère de déplacer l'interrogation et propose d'adopter un autre point de vue en "substituant alors à la question: qu'est-ce qu'un texte littéraire? celle-ci: comment lisons-nous un texte quand nous le lisons comme littéraire? sans minimiser ni exagérer la part de subjectivité dont relève ce nous" (Aron, 1984: 21). Cette réflexion invite à reconsidérer la notion de "littérarité", à la situer tout autant dans la spécificité interne du texte que dans le regard du lecteur. Pour ce qui est de Journal intime, cet article s'est efforcé de faire ressortir le système de procédés mis en œuvre par l'auteure pour montrer la spécificité de son récit et les enjeux de la conception de l'écriture du moi érotique que défend Lydia Vázquez, autant de questions à travers lesquelles se reflète une marque ou une trace de littérarité dans la mesure où elles interpellent l'expérience du lecteur, sa sensibilité ou son mode d'appréhender le monde.

\section{Conclusion}

Dans son essai au titre très évocateur, De l'infini au zéro. Anthologie historique des lectures érotiques 1985-2000, l'écrivain Jean-Jacques Pauvert dresse un bilan négatif des métamorphoses qu'a connues l'érotisme dans la littérature de langue française à la fin du $\mathrm{XX}^{\mathrm{e}}$ siècle. Le spécialiste de la littérature érotique entrevoit pour le genre un futur sombre, caractérisé par ce qu'il désigne comme la "gadgétisation du corps" ou la "désexualisation du corps féminin" (Pauvert, 2001: 693). Cependant, force est de constater que les deux premières décennies ont vu émerger une nouvelle génération d'écrivaines qui se sont approprié un espace sur la scène littéraire, en affirmant le corps féminin à travers de nouveaux codes, esthétiques et poétiques. Le domaine de la littérature érotique contemporaine qui est l'œuvre de femmes est désormais devenu un lieu d'expression et de revendication ainsi qu'un champ de recherches foisonnant, comme en témoigne l'essai d'Alexandra Destais, Éros au féminin (2014).

Avec Journal intime, Lydia Vázquez impose sa voix féminine singulière, aux modalités discursives nouvelles, et elle interroge les possibilités, à toujours poursuivre, entre les écritures du moi et la littérature érotique. Dans son récit, l'auteure déploie une langue érogène très riche et qu'elle réinvente parfois pour changer de sexe, créant ainsi des jeux de

n'est pas la littérature mais la littérarité, c'est-à-dire ce qui fait d'une œuvre donnée une œuvre littéraire" (Jakobson, 1973: 15).

13 "Quand nous parlerons de littérature et de littérarité, il ne s'agira jamais que de notions relatives et révisables. Nous ne chercherons pas pour chacune de nos propositions à vérifier si elles peuvent être valables pour tout texte littéraire, encore moins à savoir si telle ou telle propriété est capable de discriminer un texte littéraire d'un autre texte: ces questions n'ont pas de sens" (Aron, 1984: 21). 
transposition érotique qui permettent à la femme d'atteindre des sommets d'extase inédits. Ces glissements ludiques qui motivent l'érotisme féminin évoquent les inversions qui sont au cœur des plus grandes œuvres érotiques, comme celle de la poétesse Joyce Mansour.

Il convient de souligner enfin que Sade, Jarry, Pierre Louÿs sont autant d'auteurs présents dans les pages de Journal intime et dont la lecture a forgé la nature et la personnalité érotique de Lydia Vázquez. Grâce à Journal intime, l'auteure espagnole s'affirme dans le paysage de la littérature érotique contemporaine de langue française. De par sa complexité, son originalité discursive et la singularité du moi érotique féminin qu'il met en scène, Journal intime est une œuvre d'une rare beauté et qui invite à réfléchir à la place que joue la langue de l'Autre au sein de l'écriture du moi érotique.

\section{Références bibliographiques}

Allamand, Carole. 2018. Le "Pacte" de Philippe Lejeune ou l'autobiographie en théorie. Paris, Honoré Champion éditeur (coll. Textes critiques français).

Aron, Thomas. 1984. Littérature et littérarité, un essai de mise au point. Paris, Les Belles Lettres.

Bataille, Georges. 2001. Histoire de l'œil (1928). Paris, Gallimard.

Bataille, Georges. 2001. L'Érotisme (1957). Paris, Argument.

Braud, Michel. 2006. La forme des jours. Pour une poétique du journal personnel. Paris, Seuil (coll. Poétique).

Colonna, Vincent. 1989. Autofiction, essai sur la fictionnalisation de soi en littérature. Thèse de Doctorat, École des Hautes Études en Sciences Sociales.

Courbet, David. 2012. Féminismes et pornographie. Paris, La Musardine (coll. L'attrape-corps).

Deforges, Régine. 1996. L’Orage. Paris, Éditions Blanches.

Destais, Alexandra. 2014. Éros au féminin. Paris, Klincksieck.

Dubrovsky, Serge. 1977. Fils. Paris, Éditions Galilée.

FrANC, Isabel. 1992. Entre todas las mujeres. Barcelona, Tusquets editores.

Gassel, Nathalie. 2004. Stratégie d'une passion. Avin/Hannut, Éditions Luce Wilquin.

Gassel, Nathalie., 2005. Construction d'un corps pornographique. Bruxelles, Éditions Cercle d'art (coll. Éthique esthétique).

Gassel, Nathalie. 2008. Récit plastique. Liège, Éditions du Somnambule Équivoque (coll. Fulgurances). 
Anales de Filología Francesa, n. $^{\circ} 28,2020$

Mise EN SCÈNE DU MOI ÉROTIQUE, VARIATIONS AUTOBIOGRAPHIQUES ET JEUX LITTÉRAIRES DANS...

Goulemot, Jean-Marie. 1999. "Préface" in De l'obscène et de la pornographie comme objets d'études, coll. "Cahiers d'histoire culturelle", n'5, 3-9.

Jakobson, Roman. 1973. Questions de poétique. Paris, Seuil.

Lecarme, Jacques et Lecarme-Tabone, Éliane. 2015. L'Autobiographie (1997). Paris, Armand Colin.

LeJeune, Philippe. 1998. L'autobiographie en France (1971). Paris, Armand Colin.

LeJeune, Philippe. 1996. Le Pacte autobiographique (1975). Paris, Seuil.

LeJeune, Philippe. 1989. Cher cahier... Paris, Gallimard.

Lejeune, Philippe et Bogaert, Catherine. 2006. Le journal intime, histoire et anthologie. Paris, Textuel.

LeJeune, Philippe. 2015. Écrire sa vie. Du pacte au patrimoine autobiographique. Paris, Éditions du Mauconduit.

Maingueneau, Dominique. 2007. La littérature pornographique. Paris, Armand Colin.

Mansour, Joyce. 2014. Euvres complètes, prose \& poésie. París, Éditions Michel de Maule.

Millet, Catherine. 2001. La Vie sexuelle de Catherine M. Paris, Éditions du Seuil.

PAuvert, Jean-Jacques. 2000. La littérature érotique. Paris, Flammarion.

Reuter, Yves. 1996. Introduction à l'analyse du roman (1991). Paris, Dunod.

Rey, Françoise. 1989. La Femme de papier. Paris, Ramsay.

REY, Françoise. 2002. La Gourgandine. Paris, Albin Michel.

Rey, Françoise. 2002. Souvenirs lamentables. Paris, Le Cercle.

REY, Françoise. 2004. Vers les hommes. Paris, Albin Michel.

Reyes, Alina. 1988. Le Boucher. Paris, Le Seuil.

Simonet-Tenant, Françoise. 2001. Le journal intime. Paris, Nathan université.

Trekker, Annemarie et Peigny, Réjane. 2011. Écritures de l'intime. Le récit de soi face au regard de l'autre. Tellin, Éditions Traces de vie.

VÁzQuez Lydia. 2019. Journal intime. Paris, Éditions Du Murmure. 